MATHEMATICS OF COMPUTATION

Volume 71, Number 237, Pages 449-452

S $0025-5718(01) 01321-7$

Article electronically published on May 11, 2001

\title{
FIFTEEN CONSECUTIVE INTEGERS WITH EXACTLY FOUR PRIME FACTORS
}

\author{
TONY FORBES
}

\begin{abstract}
We describe a successful search for a sequence of fifteen consecutive integers, each the product of exactly four prime factors. Fifteen is best possible.
\end{abstract}

\section{INTRODUCTION}

Denote by $d(n)$ the number of divisors of $n$ and by $\Omega(n)$ the number of prime divisors of $n$, each counted according to multiplicity.

Section B18 of Richard Guy's book [2] discusses solutions of $d(n)=d(n+1)=$ $\cdots=d(n+k-1)=m$ for given $m$ and $k$. Guy states that sequences of seven, eight and nine consecutive integers with 48 divisors were found in 1990 by Ivo Duntsch and Roger Eggleton. The sequence of nine begins with 17796126877482329126044.

We consider a similar problem, with $d(n)$ replaced by $\Omega(n)$ : Given $m, k>1$, find $k$ consecutive integers which all have the same $\Omega$ value, $m$.

In the case $k=2$, the interesting question as to whether $\Omega(n)=\Omega(n+1)$ infinitely often was resolved in 1984 by Heath-Brown [3]. Heath-Brown's paper, building upon the work of Claudia Spiro [4, proved that there are infinitely many solutions of $d(n)=d(n+1)$ but it is clear from the construction used in the proof that the same is true for $\Omega(n)$.

\section{Discussion}

There is an obvious upper bound to the possible number of consecutive integers with the same $\Omega$-value, $m$, and it is interesting to discover examples of sequences where the maximum length is actually attained.

A sequence of $2^{m}$ consecutive integers must include a multiple of $2^{m}$, and $\Omega\left(q 2^{m}\right)>m$ unless $q=1$. However, we can ignore the case where the sequence includes $2^{m}$ itself, for then it must also contain at least one of $2^{m-1}$ and $5 \cdot 2^{m-2}$. Hence there can be no more than $2^{m}-1$ consecutive integers $x$ with $\Omega(x)=m$.

The Prime $k$-tuple Conjecture of Dickson [1] implies that the bound $2^{m}-1$ is attained infinitely often. Let $p_{i}, i=1,2, \ldots, 2^{m}-1, \Omega(i)<m-1$, be distinct primes greater than $2^{m}$. Define $q_{i}=p_{i}{ }^{m-1-\Omega(i)}$ if $\Omega(i)<m-1, q_{i}=1$ otherwise. Suppose $n \equiv 0(\bmod M)$, where

$$
M=\operatorname{lcm}\left(1,2, \ldots, 2^{m}-1\right) \prod_{p<2^{m}, p \text { prime }} p,
$$

Received by the editor March 14, 2000.

2000 Mathematics Subject Classification. Primary 11A51.

Key words and phrases. Prime factors. 
and $n \equiv-i\left(\bmod q_{i}\right), 1 \leq i \leq 2^{m}-1$. Then, since $\Omega(i) \leq m-1$ for $1 \leq i \leq 2^{m}-1$, the set

$$
\left\{\frac{n+i}{i q_{i}}: i=1,2, \ldots, 2^{m}-1\right\}
$$

consists of integers and furthermore it satisfies the necessary condidtion of [1] for representing $2^{m}-1$ primes.

When $m=2$ and $m=3$, finding sequences of maximum length is easy. The smallest example with $m=2$ is $\{33,34,35\}$ and for $m=3(k=7)$ it occurs at $n=211673$ :

$$
\begin{aligned}
n & =211673=7 \cdot 11 \cdot 2749 \\
n+1 & =211674=2 \cdot 3 \cdot 35279 \\
n+2 & =211675=5 \cdot 5 \cdot 8467 \\
n+3 & =211676=2 \cdot 2 \cdot 52919 \\
n+4 & =211677=3 \cdot 37 \cdot 1907 \\
n+5 & =211678=2 \cdot 109 \cdot 971 \\
n+6 & =211679=13 \cdot 19 \cdot 857
\end{aligned}
$$

If $m>4$, it is not so easy. A search for 31 consecutive integers with the same $\Omega$ value of 5 seems to be quite a difficult task, as yet unaccomplished.

For the remaining case, we exhibit here what we believe to be the only known example of a maximum-length sequence with $m=4$. The first number is

$$
n=488995430567765317569,
$$

and for each of the fifteen integers $n+i, i=0,1,2, \ldots, 14, \Omega(n+i)=4$, as can be seen from the factorizations:

$$
\begin{aligned}
n & =3 \cdot 3 \cdot 3 \cdot 18110941872880196947 \\
n+1 & =2 \cdot 5 \cdot 11 \cdot 4445413005161502887 \\
n+2 & =6917 \cdot 19973 \cdot 130843 \cdot 27051617 \\
n+3 & =2 \cdot 2 \cdot 3 \cdot 40749619213980443131 \\
n+4 & =13 \cdot 17 \cdot 283 \cdot 7818547728247211 \\
n+5 & =2 \cdot 7 \cdot 7 \cdot 4989749291507809363 \\
n+6 & =3 \cdot 5 \cdot 5 \cdot 6519939074236870901 \\
n+7 & =2 \cdot 2 \cdot 2 \cdot 61124428820970664697 \\
n+8 & =149 \cdot 28229 \cdot 4622647 \cdot 25149671 \\
n+9 & =2 \cdot 3 \cdot 3 \cdot 27166412809320295421 \\
n+10 & =31 \cdot 2963 \cdot 34871 \cdot 152667661633 \\
n+11 & =2 \cdot 2 \cdot 5 \cdot 24449771528388265879 \\
n+12 & =3 \cdot 7 \cdot 11 \cdot 2116863335791191851 \\
n+13 & =2 \cdot 37 \cdot 922213309 \cdot 7165420727 \\
n+14 & =19 \cdot 29 \cdot 60607 \cdot 14643011879719
\end{aligned}
$$


Our strategy is to fix $n$ modulo the product of some small prime powers so that nine of the numbers are each divisible by exactly three small primes. Then we perform a nine-way sieving operation on the cofactors. The aim is to produce nine primes and hence nine numbers that have four prime factors. Each time we find nine primes we analyse the remaining six numbers to see if, by chance, they are products of exactly four primes.

Write

$$
B_{0}=\{0,1,3,5,6,7,9,11,12\}
$$

and

$$
B_{1}=\{2,4,8,10,13,14\}
$$

Consider the fifteen numbers $N+b, b=0,1, \ldots, 14$. We fix the divisors of $N+i$, $i \in B_{0}$, thus:

$$
\begin{aligned}
N & =3^{3} \cdot P_{0} \\
N+1 & =2 \cdot 5 \cdot 11 \cdot P_{1} \\
N+3 & =2^{2} \cdot 3 \cdot P_{3} \\
N+5 & =2 \cdot 7^{2} \cdot P_{5} \\
N+6 & =3 \cdot 5^{2} \cdot P_{6} \\
N+7 & =2^{3} \cdot P_{7} \\
N+9 & =2 \cdot 3^{2} \cdot P_{9} \\
N+11 & =2^{2} \cdot 5 \cdot P_{11} \\
N+12 & =3 \cdot 7 \cdot 11 \cdot P_{12} .
\end{aligned}
$$

Let

$$
N=j Q+H
$$

where $Q=2^{4} \cdot 3^{4} \cdot 5^{2} \cdot 7^{2} \cdot 11=17463600$ and $H=13363569$. Observe that $H \equiv 27$ $(\bmod 81), H \equiv-1(\bmod 11), H \equiv-5(\bmod 49), H \equiv-6(\bmod 25)$ and $H \equiv 1$ $(\bmod 16)$.

We perform a 9 -way sieve of the numbers $j$ by primes up to a suitable limit. That is, we start with a list of numbers $j=0,1,2, \ldots$ and for each sieving prime $p$ we remove all those $j$ for which $(j Q+H+b) / e_{b} \equiv 0(\bmod p)$ for at least one $b \in B_{0}$. Here, $e_{b}$ is the product of the fixed prime divisors of $N+b$.

For surviving $j$ 's we check to see if $(j Q+H+b) / e_{b}$ is prime for all $b \in B_{0}$. Each time we find nine primes we factorize the remaining six numbers $N+b, b \in B_{1}$, to see if they too are products of four primes. Letting $j$ run from 0 to 28314132275200 we found 12590 sets of nine primes, each producing a set of numbers $N+b, b \in B_{0}$, with $\Omega(N+b)=4$. Our analysis of the sets of $N+b, b \in B_{1}$, produced a total of 18942 numbers with four prime factors, broken down as in Table 1. For the expected frequency we assume a binomial distribution with parameter $\frac{18942}{6 \cdot 12590}=0.25075$ approximately. 
TABLE 1.

\begin{tabular}{|c|r|r|r|}
\hline $\begin{array}{c}\Omega(N+b)=4, \\
b \in B_{1}\end{array}$ & frequency & weighted & expected \\
\hline 0 & 2168 & 0 & 2227.3 \\
1 & 4520 & 4520 & 4472.5 \\
2 & 3800 & 7600 & 3742.1 \\
3 & 1642 & 4926 & 1669.8 \\
4 & 405 & 1620 & 419.1 \\
5 & 54 & 270 & 56.1 \\
6 & 1 & 6 & 3.1 \\
\hline & 12590 & 18942 & 12590.0 \\
\hline
\end{tabular}

\section{REFERENCES}

1. L. E. Dickson, A new extension of Dirichlet's theorem on prime numbers, Messenger of Mathematics 33 (1904), 155-161.

2. Richard K. Guy, Unsolved Problems in Number Theory, 2nd ed., Springer-Verlag, New York, 1994. MR 96e: 11002

3. D. R. Heath-Brown, The divisor function at consecutive integers, Mathematika 31 (1984), 141-149. MR 86c:11071

4. C. Spiro, Thesis, Urbana, 1981.

22 St. Albans Road, Kingston upon Thames, Surrey, KT2 5HQ, England

E-mail address: tonyforbes@ltkz.demon.co.uk 